\title{
Bio-based epoxidized starch wood adhesives: the effect of amylopectin and amylose content on adhesion properties
}

\author{
Nicole Tratnik, ${ }^{\text {a }}$ Pei-Yu Kuo,b Nicolas R. Tanguy,c Pitchaimari Gnanasekar, ${ }^{\mathrm{c}}$ and Ning Yan ${ }^{\mathrm{a}, \mathrm{c}^{*}}$ \\ a University of Toronto, Graduate Department of Forestry, 33 Willcocks Street, Toronto, ON M5S 3B3, Canada. \\ ${ }^{\mathrm{b}}$ National Ilan University, Department of Forestry and Natural Resources, 1 Shennong road, Yilan City, 26047, Taiwan \\ c University of Toronto, Department of Chemical Engineering and Applied Chemistry, 200 College Street, Toronto, ON \\ M5S 3E5, Canada. ${ }^{1}$ \\ *ning.yan@utoronto.ca
}

Number of pages: 12

Number of tables: 5

Number of figures: 5 


\section{Epoxy Equivalent Weight (EEW)}

Epoxy equivalent weight (EEW) was measured using the ASTM D 1652 method. Using manual titration, 0.1 N Perchloric Acid was standardized with $0.25-0.40 \mathrm{~g}$ of potassium hydrogen phthalate three times. Samples of epoxidized starch adhesive weighing approximately $0.4 \mathrm{~g}$ were dissolved in $10-15 \mathrm{~mL}$ methylene chloride and $10 \mathrm{~mL}$ tetraethyl ammonium bromide reagent. Crystal violet was used as an indicator. The solution was titrated with perchloric acid to the end point which was when the solution changed from blue to green. All samples were tested in triplicate.

The EEW values in Table 2 were calculated to determine the amount of hardener needed to be fully reacted as an adhesive. The EEW value of epoxidized high amylose starch is slightly higher than the epoxidized low amylose and amylopectin starch which indicated a lower degree of substitution consistent with the NMR data. The combined evidence from the FTIR spectrum, NMR spectrum and EEW values confirms successful epoxidation of starch. 
Table S1 EEW Values of epoxidized starches and DGEBA

\begin{tabular}{ll}
\hline & EEW (g/mol) \\
\hline Epoxidized High Amylose & 358 \\
\hline Epoxidized Low Amylose & 359 \\
\hline Epoxidized Amylopectin & 364 \\
\hline DGEBA & 171 \\
\hline
\end{tabular}


Table S2 Starch Characterization

\begin{tabular}{llll}
\hline & $\begin{array}{l}\text { Amylose } \\
\text { Content } \\
(\%)\end{array}$ & $\begin{array}{l}\text { Amylopectin } \\
\text { Content } \\
(\%)\end{array}$ & $\begin{array}{l}\text { Crystallinity } \\
(\%)\end{array}$ \\
\hline High Amylose & $71 \pm 2.6$ & $29 \pm 2.6$ & $16 \%$ \\
\hline Low Amylose & $36 \pm 0.4$ & $64 \pm 0.4$ & $23 \%$ \\
\hline Amylopectin & $0 \pm 1.0$ & $100 \pm 1.0$ & $50 \%$ \\
\hline
\end{tabular}




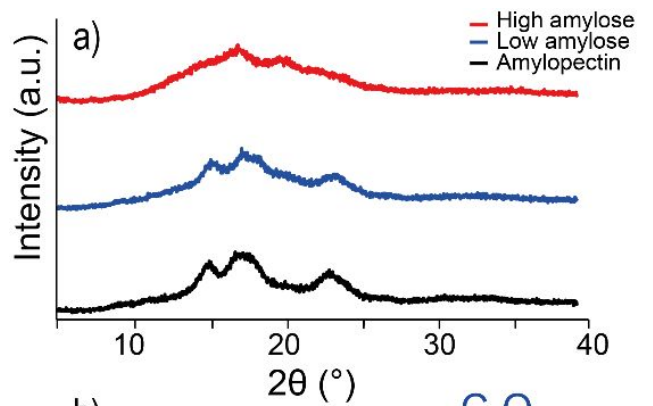

c)

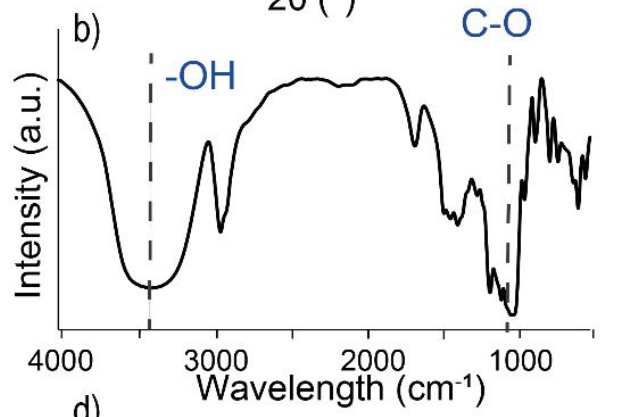

d)

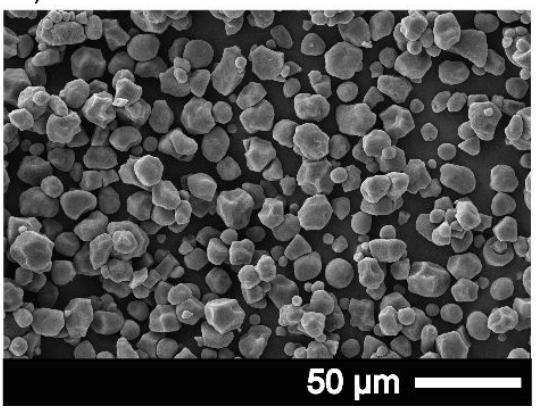

e)

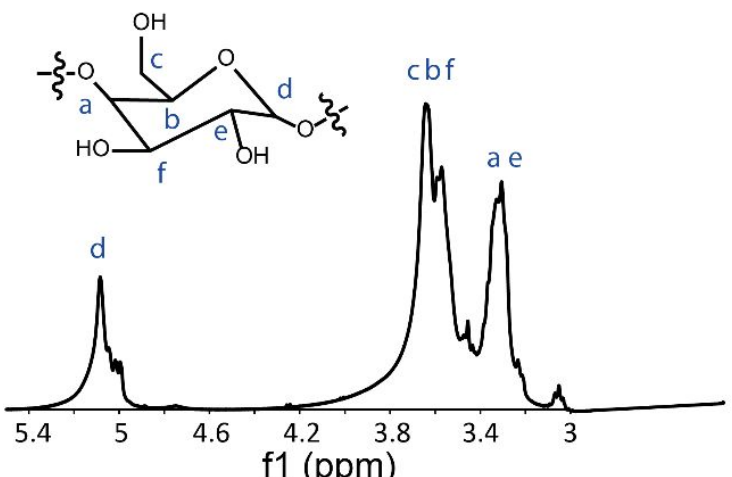

DMSO
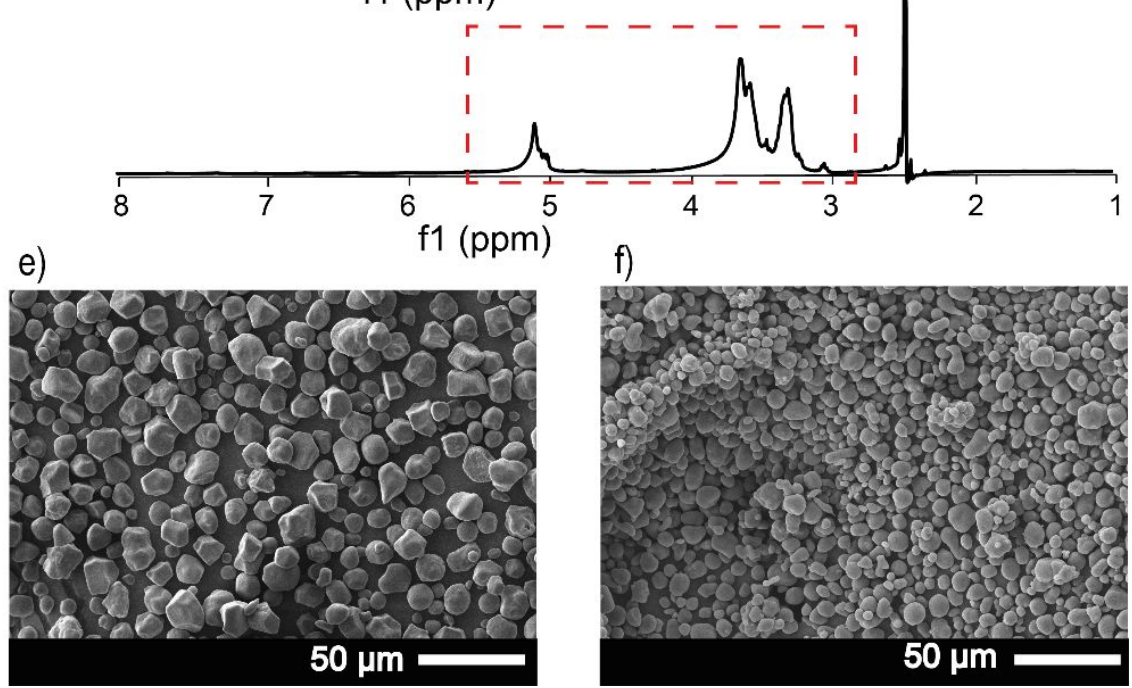

Figure S1. a) X-ray diffraction spectra of the three starches, b) FTIR spectra of high amylose starch, c) NMR spectra of high amylose starch; SEM images of d) amylopectin, e) low amylose, f) high amylose starches. 


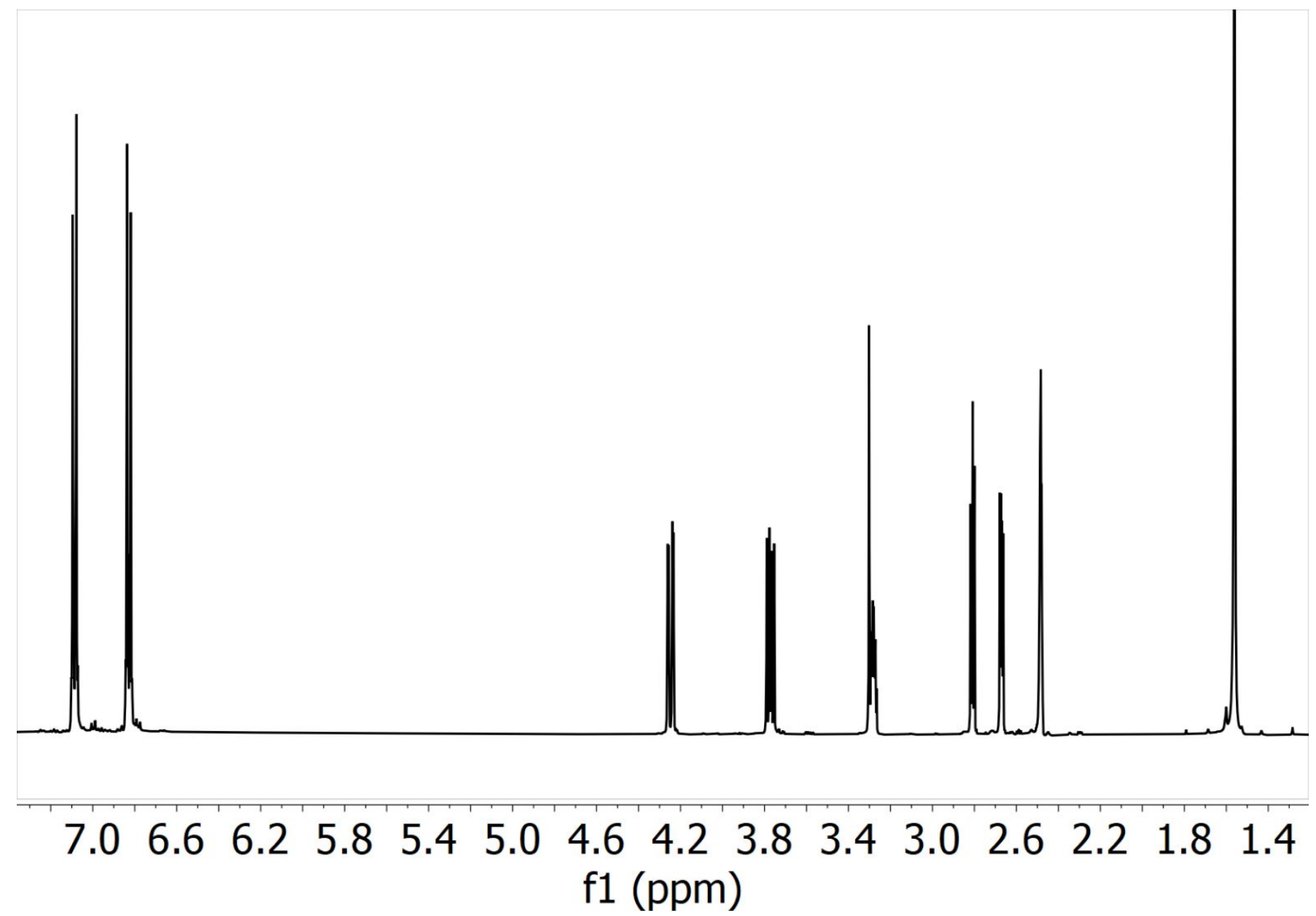

Figure S2 ${ }^{1} \mathrm{H}$ NMR of DGEBA 
a)

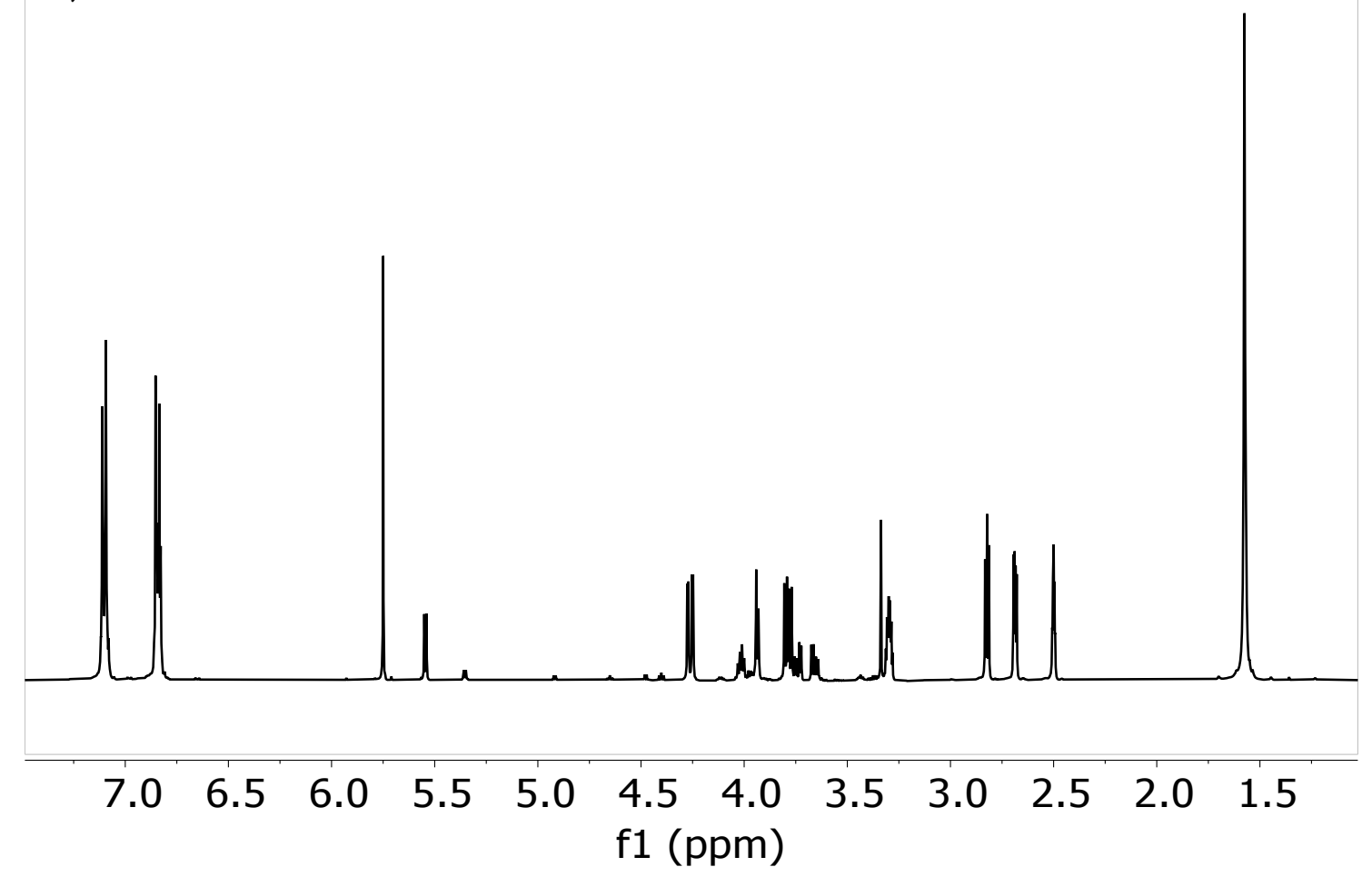

b)

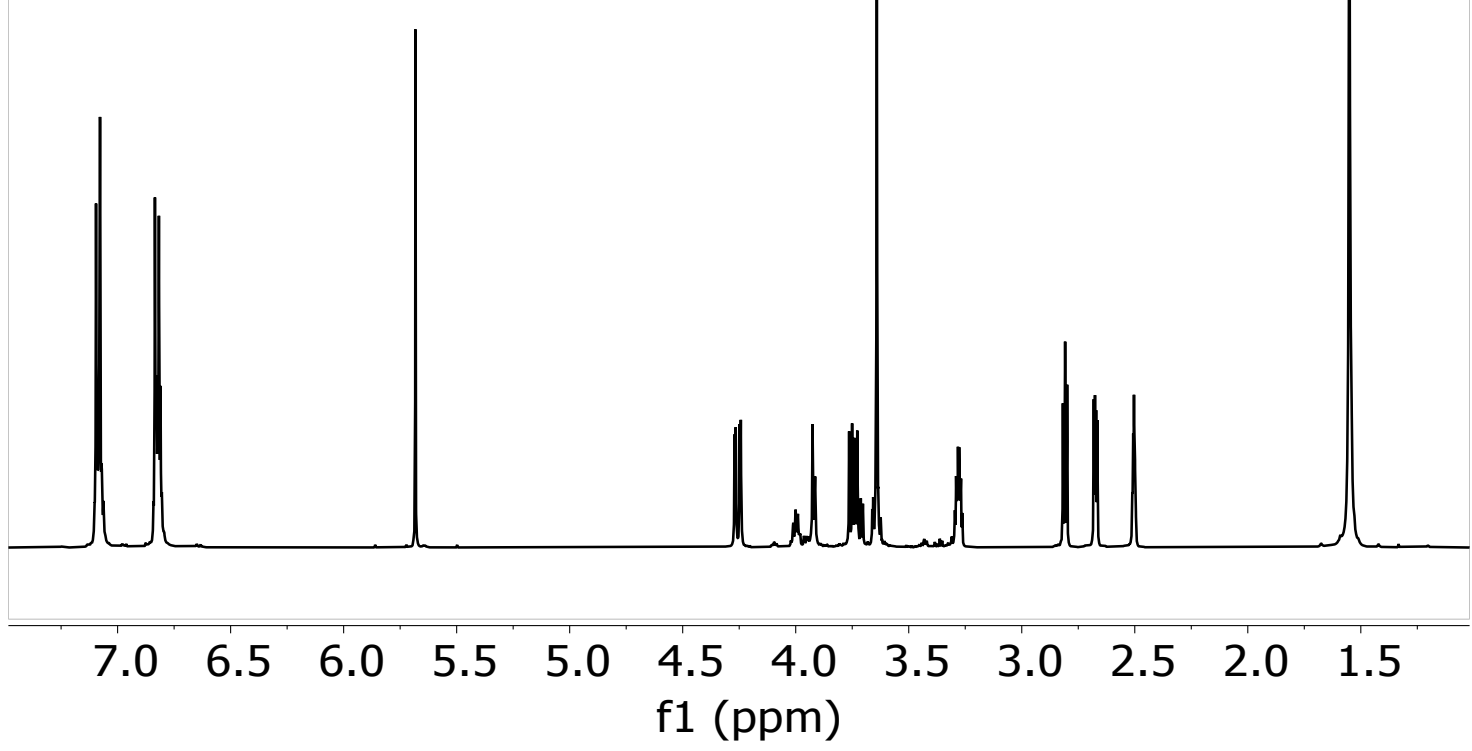

Figure S3 ${ }^{1} \mathrm{H}$ NMR of a) E-high amylose starch in dmso-d6 b) E-high amylose starch in dmso-d6 with a drop of $\mathrm{D}_{2} \mathrm{O}$ added 
Table S3 FT-IR Band Assignments

\begin{tabular}{ll}
\hline Wavenumber $\left(\mathbf{c m}^{-1}\right)$ & Assignment \\
\hline Starch & \\
\hline $\mathbf{3 0 4 0 - 3 8 3 0}$ & O - H stretching \\
\hline $\mathbf{3 0 0 0 - 2 8 5 0}$ & C - H vibrations \\
\hline $\mathbf{1 6 6 8}$ & Bound Water \\
\hline $\mathbf{1 1 7 0}$ & C - O - C asymmetric stretch \\
\hline $\mathbf{1 0 7 9 , 1 0 1 3}$ & C - O vibration (anhydroglucose ring) \\
\hline $\mathbf{9 2 5 , 7 5 8}$ & Entire anhydroglucose ring stretching \\
\hline $\mathbf{E p 0 x y}$ & \\
\hline $\mathbf{3 0 5 5 , 1 6 0 8}$ & Aromatic C = C vibrations \\
\hline $\mathbf{2 9 5 0 - 2 8 5 0}$ & C - H vibrations \\
\hline $\mathbf{1 1 6 8}$ & C - O vibrations \\
\hline $\mathbf{1 0 3 4}$ & Ar - O - C stretching \\
\hline $\mathbf{9 1 5}$ & Oxirane ring asymmetric vibrations \\
\hline
\end{tabular}


Table S4 NMR assignments

\begin{tabular}{|c|c|c|}
\hline Molecule & Proton & Chemical Shift \\
\hline \multirow[t]{6}{*}{ Epoxy } & & $2.80,2.67$ \\
\hline & & 3.27 \\
\hline & & $4.24,3.73$ \\
\hline & & 7.10 \\
\hline & & 6.83 \\
\hline & & 1.55 \\
\hline \multirow[t]{6}{*}{ Starch } & & 3.73 \\
\hline & & 3.73 \\
\hline & & 3.73 \\
\hline & & 3.73 \\
\hline & & 3.73 \\
\hline & & 5.68 \\
\hline \multirow{3}{*}{$\begin{array}{l}\text { Epoxy } \\
\text { opening }\end{array}$} & is & 3.93 \\
\hline & Oivs & 4.01 \\
\hline & Oiver & 3.03 \\
\hline
\end{tabular}


Table S5 DSC results for blended starches

\begin{tabular}{|c|c|c|c|c|c|c|c|c|c|}
\hline $\begin{array}{l}\text { Resin } \\
\text { Type }\end{array}$ & Sample & $\begin{array}{l}\text { Heating } \\
\text { rate }(\beta) \\
{ }^{\circ} \mathrm{C} / \mathrm{min}\end{array}$ & $\begin{array}{r}\mathrm{T}_{\mathrm{S}} \\
\left({ }^{\circ} \mathrm{C}\right)\end{array}$ & $\begin{array}{l}\mathrm{T}_{\mathrm{MAX}} \\
\left({ }^{\circ} \mathrm{C}\right)\end{array}$ & $\begin{array}{l}\mathrm{T}_{\mathrm{E}} \\
\left({ }^{\circ} \mathrm{C}\right)\end{array}$ & $\begin{array}{l}\mathrm{T}_{\mathrm{E}}-\mathrm{T}_{\mathrm{S}} \\
\left({ }^{\circ} \mathrm{C}\right)\end{array}$ & $\begin{array}{l}\Delta \mathrm{H}_{\mathrm{C}} \\
\left(\mathrm{kJmol} \mathrm{m}^{-1}\right)\end{array}$ & $\begin{array}{l}\mathrm{Tg} \\
\left({ }^{\circ} \mathrm{C}\right) \\
@ \\
{ }^{\circ} \mathrm{C} / \mathrm{min}\end{array}$ & 10 \\
\hline \multirow{3}{*}{ 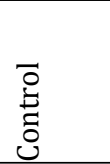 } & \multirow{3}{*}{ DGEBA } & 5 & 49 & 78 & 104 & 55 & 98 & \multirow{3}{*}{117} & \\
\hline & & 10 & 58 & 92 & 124 & 66 & 106 & & \\
\hline & & 15 & 65 & 101 & 136 & 71 & 180 & & \\
\hline \multirow{9}{*}{ 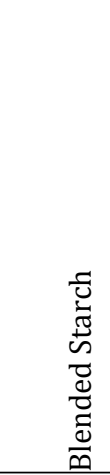 } & \multirow{3}{*}{ High Amylose } & 5 & 49 & 79 & 108 & 59 & 112 & \multirow{3}{*}{113} & \\
\hline & & 10 & 57 & 91 & 124 & 67 & 116 & & \\
\hline & & 15 & 63 & 104 & 144 & 81 & 90 & & \\
\hline & \multirow{3}{*}{ Low Amylose } & 5 & 44 & 77 & 110 & 66 & 192 & \multirow{3}{*}{115} & \\
\hline & & 10 & 60 & 91 & 124 & 64 & 211 & & \\
\hline & & 15 & 67 & 102 & 137 & 70 & 215 & & \\
\hline & \multirow{3}{*}{ Amylopectin } & 5 & 46 & 79 & 106 & 60 & 100 & \multirow{3}{*}{117} & \\
\hline & & 10 & 59 & 92 & 124 & 65 & 116 & & \\
\hline & & 15 & 67 & 103 & 140 & 73 & 102 & & \\
\hline
\end{tabular}




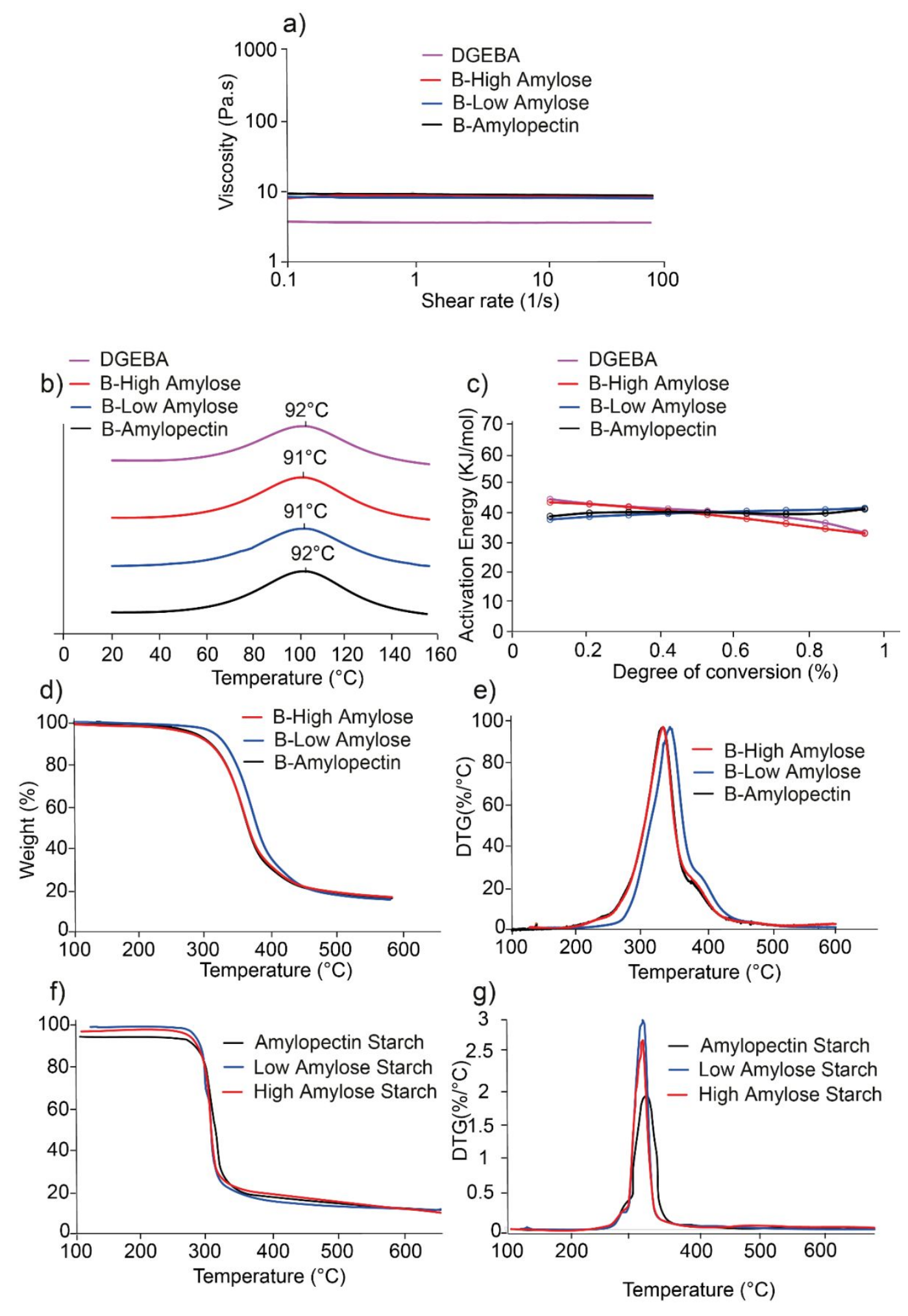

Figure S4 a) Viscosities of blended starches b) DSC measurements of blended starches c) Activation energies of blended starches in relation to degree of conversion d) TGA graph of blended starches e) DTG graph of blended starched e) TGA graph of native starches f) DTG graph of native starched 

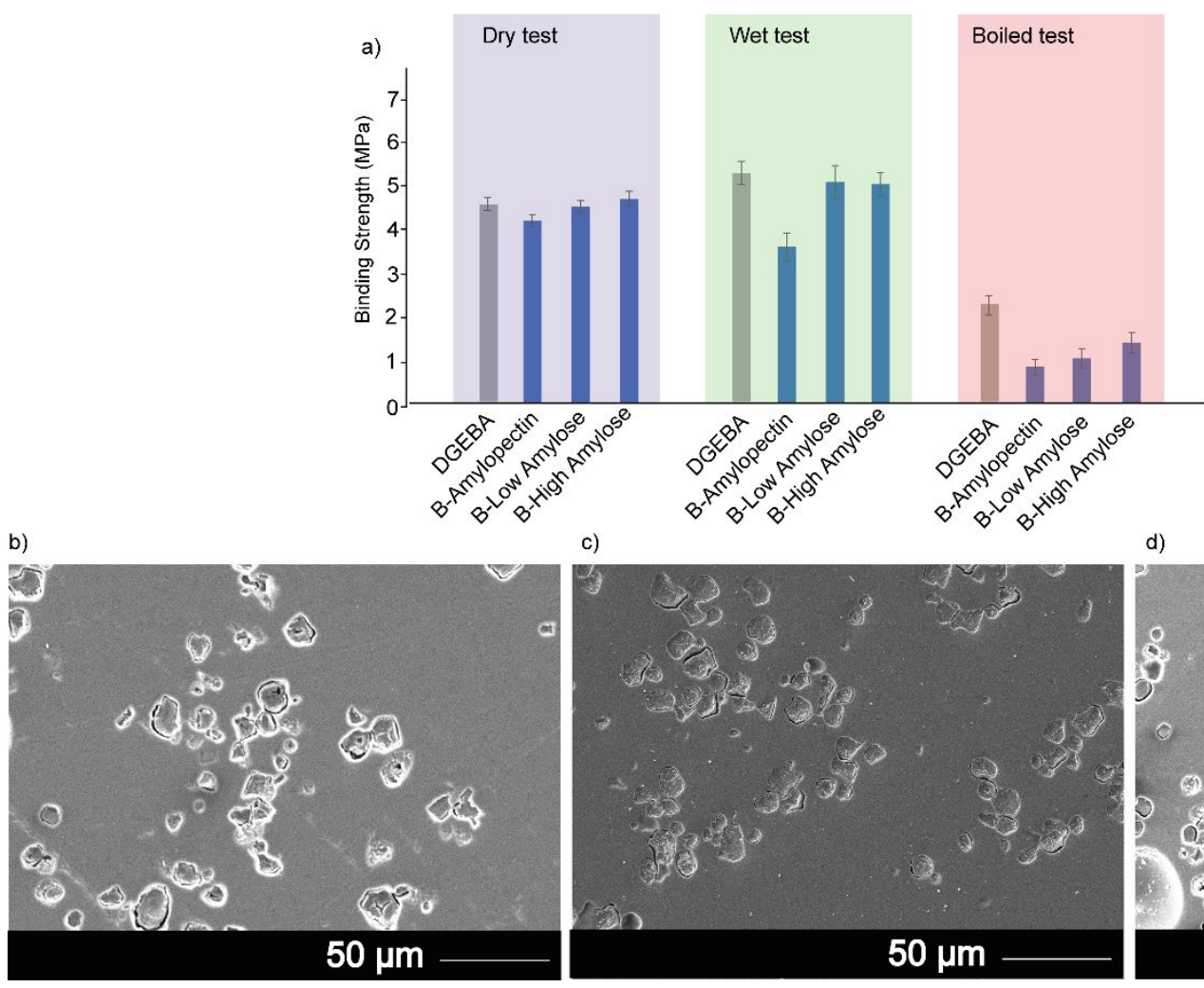

c)

d)

Figure S5 a) Bonding strength of blended starches, and SEM of b)B-amylopectin c)B-low amylose and d) B-high amylose cured starches 